\title{
Elementare Beweise des Satzes von Morley
}

\author{
Gerhard Wanner \\ Gerhard Wanner, geboren 1942 in Innsbruck, unterrichtet seit 1973 an der Universität \\ Genf. Sein hauptsächliches Arbeitsgebiet (in enger Zusammenarbeit mit Ernst Hairer) \\ ist die numerische Lösung von Differentialgleichungen. Darüber hinaus gibt er auch \\ gerne Einführungsvorlesungen über Analysis und Geometrie.
}

Une démonstration élémentaire de ce théorème n'est pas facile.

(J. Marchand, L'Ens. Math. XXIX (1930), p. 291)

La méthode de Morley ressortissant à une géométrie analytique du plan de la variable complexe, on s'empressa ... de rechercher une démonstration aussi courte et aussi élégante que l'énoncé ... A mon avis, de tels désirs ne sauraient être satisfaits.

(H. Lebesgue, L'Ens. Math. XXXVIII (1939), p. 39)

Morley's theorem is startling, difficult to prove, and utterly beautiful.

(W. Dunham, Euler, the master of us all. Math. Ass. Amer. 1999)

... pour apprécier le théorème, il est bon d'essayer soi-même des démonstrations géométriques, ou même trigonométriques, avant de lire ce qui suit.

(M. Berger, Géométrie I, 1990)

Der elegante Satz von Morley über die Winkeldreiteilenden eines Dreiecks entwickelt sich langsam zu einem „,must“ des Geometrieunterrichts. Die vorliegende Arbeit schliesst sich an den Artikel [4] der Elemente an, in welchem ein auf den komplexen Zahlen beruhender Beweis von Alain Connes vorgestellt wurde. Wir interessieren uns hier für möglichst elementare Beweise, alte und neue, für diesen Satz. Wir stossen dabei noch auf weitere prominente Namen des letzten Jahrhunderts: Henri Lebesgue, Roger Penrose und John Conway. Recht erstaunlich für einen Satz, den der Entdecker ursprünglich gar nicht veröffentlichen wollte. 


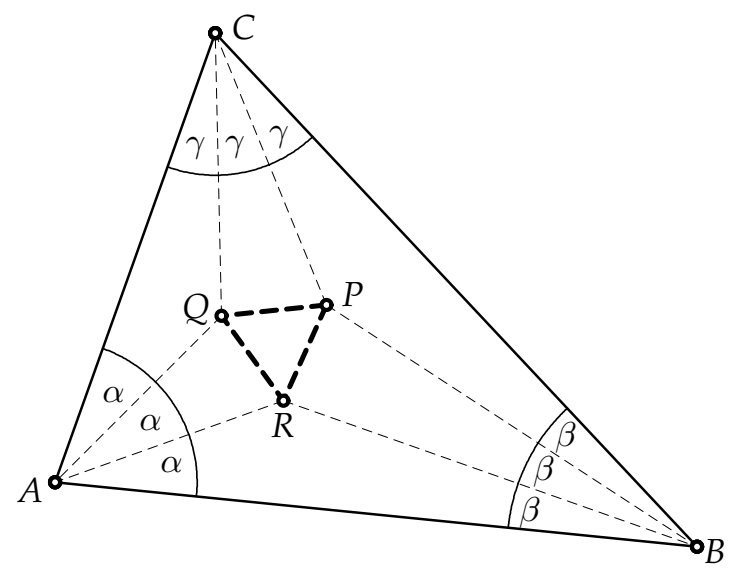

Fig. 1 Der Satz von Morley

Satz. Für jedes Dreieck $A B C$ ist das Dreieck $P Q R$, welches durch die Schnittpunkte der Winkeldreiteilenden von ABC gebildet wird (siehe Fig. 1), ein gleichseitiges Dreieck.

Dieser elegante und überraschende Satz, der inzwischen auch „Morley’s Miracle“ heisst, ist mehr als 100 Jahre alt ${ }^{1}$. Bereits die erste Veröffentlichung [7] enthält drei Beweise, von denen der dritte auf trigonometrischem Wege die Formel

$$
Q R=8 r \sin \alpha \sin \beta \sin \gamma \quad(r=\text { Umkreisradius })
$$

herleitet, welche später „Formel von Kowalewski“ heissen wird, und deren Symmetrie die Gleichheit aller drei Seiten nach sich zieht. Dieser Beweis wird das Jahrhundert hindurch der am häufigsten gegebene sein, insbesondere im oben zitierten Buch von M. Berger. Andere Beweise benützen Eigenschaften der komplexen Zahlenebene, wie zum Beispiel der Beweis von A. Connes, welcher kürzlich den Lesern der Elemente der Mathematik durch den Artikel von Hansjörg Geiges [4] vorgestellt wurde.

Der Satz hatte das 20. Jahrhundert hindurch den Mythos, nur schwer zu beweisen zu sein (siehe Zitate). Wir interessieren uns hier für die Möglichkeiten, diesen Satz möglichst elementar zu beweisen. Wir werden dabei häufig auf den Satz über die Winkelsumme im Dreieck (Euklid I.32) und auf den Satz über Zentrums- und Peripheriewinkel im Kreis (Euklid III.20) verweisen (etwa [3], Vol. I, S. 316, Vol. II, S. 46).

Der hier vorgeschlagene Beweis. Die Hauptschwierigkeit der üblichen Beweise besteht darin, dass die Form, Lage und Grösse des Dreieckes $P Q R$ völlig unbekannt ist und dass die schönen Eigenschaften eines gleichseitigen Dreieckes erst dann benützbar wären, wenn man sie nicht mehr braucht. Daher operieren die einfachsten Beweise rückwärts,

1) Die Angaben der Literatur pendeln zwischen 1899 und 1904; die erste Zahl scheint aus der Angabe ,some 14 years ago" aus [7] extrapoliert zu sein; die Jahreszahl 1904 stammt aus einem Brief von F. Morley selbst, welcher in einer Fussnote der Arbeit von G. Loria, Math. Gazette 23 (1939), S. 367, abgedruckt ist. 


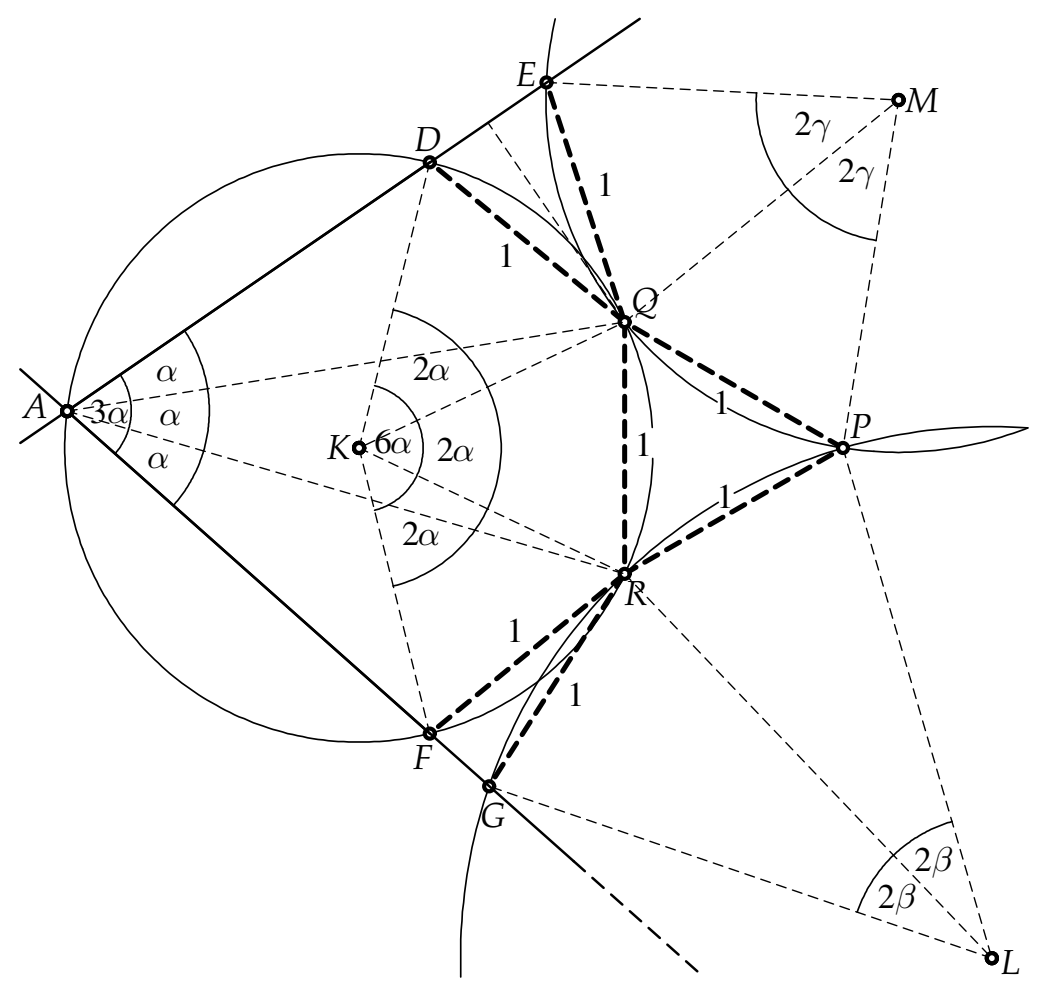

Fig. 2 Erster Beweis des Satzes von Morley

d.h. man merkt sich von dem Dreieck $A B C$ der Fig. 1 nur die Winkel $\alpha, \beta$ und $\gamma$, welche

$$
\alpha+\beta+\gamma=60^{\circ}
$$

erfüllen (Euklid I.32), plaziert ein gleichseitiges Dreieck $P Q R$ der Seitenlänge 1 nach Belieben (etwa so, dass die Seite $R Q$ senkrecht steht; siehe Fig. 2) und rekonstruiert ein Dreieck, das wir wieder $A B C$ nennen, und das zum ursprünglichen Dreieck ähnlich ist. Wegen der Eindeutigkeit des Morleyschen Dreieckes ist dieser, an und für sich, „logische Unsinn" durchaus gerechtfertigt.

Über jeder Seite dieses Dreieckes ziehen wir drei Kreise mit Zentren $K, L, M$ und Zentrumswinkel $2 \alpha, 2 \beta$ und $2 \gamma$. Dann tragen wir die vier Sekanten $Q D, Q E, R F$ und $R G$, alle auch mit der Länge 1, auf. Dadurch haben wir in jedem Kreis gleiche Zentriwinkel.

Das „Wunder“ ist nun (denn ein Wunder muss ja sein), dass der Schnittpunkt $A$ der beiden Geraden ED und GF auf der Kreisperipherie liegt. Um das zu zeigen, benützen wir, dass das Dreieck $D Q E$ gleichschenklig ist. Die Strecke $D Q$ entsteht aus der Strecke $Q R$, wenn man sie um den Winkel $2 \alpha$ um $K$ herum dreht. Deshalb bildet sie mit der positiven horizontalen Achse den Winkel $90^{\circ}+2 \alpha$. Ähnlich bildet die Strecke EQ (entstanden aus $Q P$ durch Drehung in die andere Richtung) den Winkel $150^{\circ}-2 \gamma$. Die Winkelsymmetrale DQE besitzt also den Winkel $120^{\circ}+\alpha-\gamma$ (arithmetisches 
Mittel), so dass die Gerade $D E$, welche darauf senkrecht steht, den Neigungswinkel $30^{\circ}+\alpha-\gamma$ hat. Ebenso besitzt die Strecke $F G$ einen Neigungswinkel von $30^{\circ}+\alpha-\beta$ (negativ gemessen). Somit ist der Winkel, den die Geraden $D E$ und $F G$ einschliessen (die Summe der beiden)

$$
60^{\circ}+2 \alpha-\gamma-\beta=3 \alpha,
$$

wobei (1) benutzt wurde. Da der Zentrumswinkel $F K D$ genau das Doppelte, nämlich $6 \alpha$ ist, folgt die Behauptung aus Euklid III.20, in umgekehrter Richtung angewendet. Damit sind nach Euklid III.20, jetzt in normaler Richtung angewendet, die Winkel $F A R, R A Q$ und $Q A D$ alle gleich $\alpha$.

Dieselbe Konstruktion der Punkte $B$ und $C$ liefert schliesslich das gesuchte Dreieck $A B C$ mit den gewünschten Eigenschaften.

Die Variante von Ch. Aebi (2003, pers. Mitteilung). Wenn die Punkte $A, B$ und $C$ beliebig auf den Kreisen gewählt werden, erhält man ein Neuneck $A F G B \ldots E D A$. Dann berechnet man den Winkel $\widehat{Q D E}$ mit $120^{\circ}-\alpha-\gamma=60^{\circ}+\beta$. Das Polygon $A R Q D$ ist ein Sehnenviereck, somit ergänzen sich gegenüberliegende Winkel zu $180^{\circ}$ (Euklid III.22). Es wird also der Winkel in $D$ gestreckt, falls $A$ so gewählt ist, dass auch der Winkel $\widehat{A R Q}=60^{\circ}+\beta$ ist. Das ist aber gleichbedeutend mit $\widehat{R Q A}=60^{\circ}+\gamma$, d.h. mit der Streckung des Winkels in $F$. Verfahren wir genauso bei $B$ und $C$, so wird schliesslich aus dem Neuneck ein Dreieck.

Der Beweis von J. Conway. Dieser „undisputedly simplest proof“, welcher ab 1995 im Internet verbreitet wurde (siehe http://www.cut-the-knot.com/triangle/Morley/), definiert sieben abstrakte Dreiecke über ihre Winkel wie folgt

$$
\begin{array}{ccccccc}
P Q R & A R Q & R B P & Q P C & P B C & A Q C & A B R \\
0^{*}, 0^{*}, 0^{*}, & \alpha, \beta^{*}, \gamma^{*}, & \alpha^{*}, \beta, \gamma^{*}, & \alpha^{*}, \beta^{*}, \gamma, & \alpha^{* *}, \beta, \gamma, & \alpha, \beta^{* *}, \gamma, & \alpha, \beta, \gamma^{* *},
\end{array}
$$

wobei , $x^{*}$ “ für ,,$x+60^{\circ}$ “ steht. Diese Dreiecke werden dann geschickt skaliert; das erste beliebig (etwa mit Seitenlänge 1), die nächsten drei so, dass $R Q=1, R P=$ 1 und $Q P=1$, und die letzten drei so, dass etwa $R G=1$ (wobei $R F G$ ein mit vorgeschriebenen Winkeln eingefügtes gleichschenkliges Dreieck ist). Dann wird für alle Winkel und alle Seiten nachgerechnet, dass sie schön zusammenpassen. Im Grunde genommen ist der obige Beweis zu diesem hier äquivalent, nur dass die Grössen völlig anders herauskommen.

Der Beweis von Penrose und Coxeter. Wir verlängern die Winkeldreiteilenden über die Punkte $P, Q, R$ hinaus und erhalten drei weitere Schnittpunkte $U, V, W$ (siehe Fig. 3).

Nun bestand die Hauptschwierigkeit des zweiten Beweises in [7] gerade darin, sehr mühevoll zu zeigen, dass die Dreiecke $Q W P, P V R$ und $R U Q$ gleichschenklig sind. Der Beweis von Penrose (siehe [6]), etwas vereinfacht in Coxeter [2] ${ }^{2}$, geht rückwärts von

2) Coxeter stellt Penrose, welcher bei der Abfassung dieses Beweises 22 Jahre alt war, folgendermassen vor: „Roger Penrose is a son of Professor Lionel Penrose the genetist, and a brother of Jonathan Penrose the chess champion“. Inzwischen braucht Sir Roger Penrose so eine Einführung nicht mehr. 


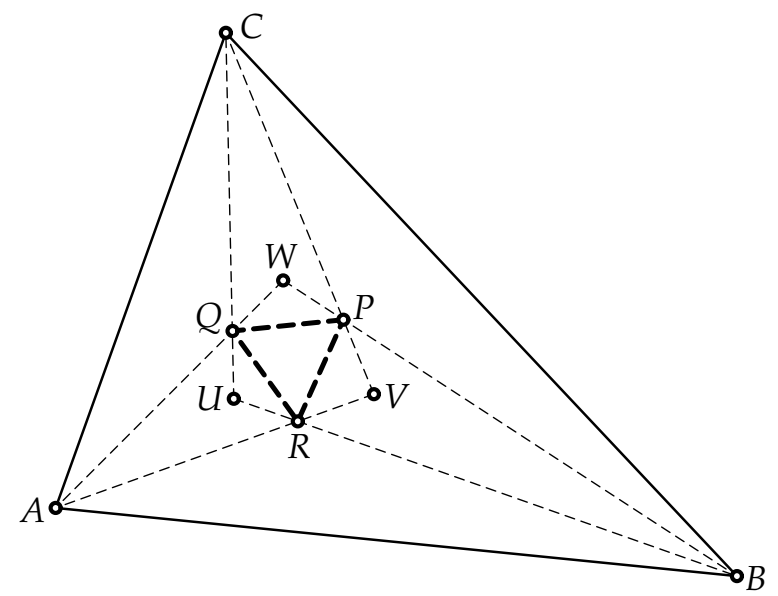

Fig. 3 Beweis von Penrose

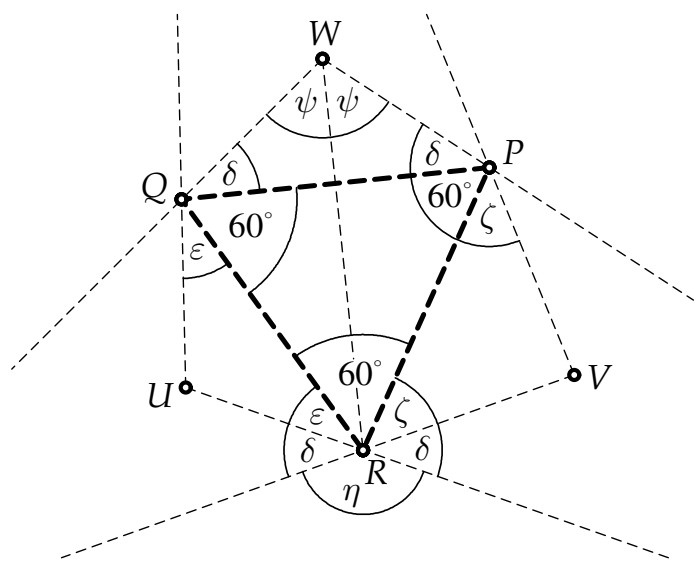

Fig. 4 Beweis von Coxeter

einem gleichseitigen Dreieck $P Q R$ aus, und setzt diesem drei gleichschenklige Dreiecke auf (siehe Fig. 4), mit beliebigen Basiswinkeln $\delta, \epsilon$ und $\zeta$, welche nur

$$
\delta+\epsilon+\zeta=120^{\circ}
$$

erfüllen sollen. Die verlängerten Scheitellinien dieser Dreiecke schneiden sich dann in den Punkten $A, B$ und $C$. Der Punkt $R$ liegt trivialerweise auf der Winkelsymmetralen von $A W B$. Wegen (3) wiederholen sich die Winkel $\delta$ um den Punkt $R$ herum. Wenn man dann die Winkel um $R$ aufsummiert, sieht man, dass $\eta=90^{\circ}+\psi$. Das ist aber gleichbedeutend damit, dass der Punkt $R$ Inkreismittelpunkt des Dreieckes $A W B$ ist (dies sieht man aus Fig. 5, wenn man Euklid I.32 auf die Dreiecke $A B W$ und $A B R$ anwendet). Also sind die unteren beiden Winkel in $A$ und in $B$ jeweils gleich gross. Wendet man das gleiche Argument rund um das Dreieck herum an, dann sieht man, dass 


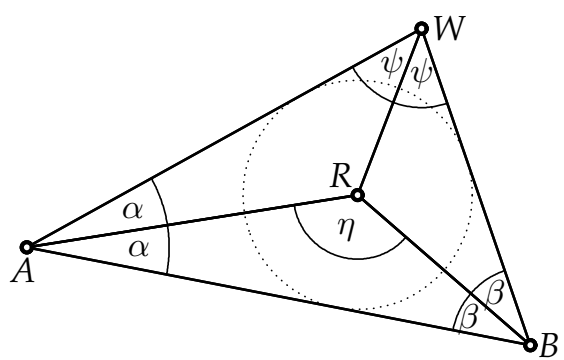

Fig. 5

überall alle drei Winkel gleich sein müssen. Weiter ist

$$
\delta=\alpha+\beta, \quad \epsilon=\beta+\gamma, \quad \zeta=\gamma+\alpha
$$

(etwa ist $\delta$ ein Aussenwinkel des Dreieckes $A B R$ ), so dass man genügend Freiheit hat, um alle Winkelkombinationen $\alpha, \beta, \gamma$, welche (1) erfüllen, auf diese Weise zu erzeugen.

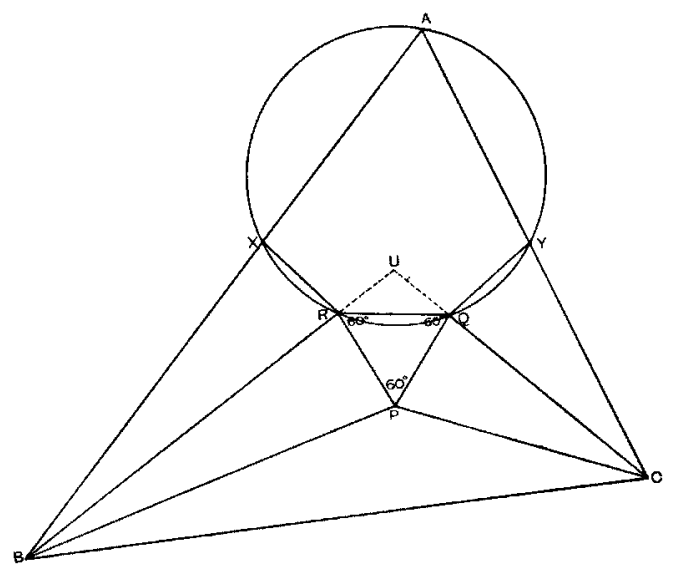

Fig. 6 Beweis von Child (kopiert aus [1])

Hybridbeweise. Zum Schluss weisen wir noch auf die drei Beweise in [1], [5] und [8] hin, welche wir „Hybridbeweise“ nennen wollen, denn sie vermischen Ideen eines Vorwärtsbeweises mit jenen eines Rückwärtsbeweises. Es wird nämlich von zwei Ecken des Dreieckes, etwa $B$ und $C$, mit seinen Winkeldreiteilenden ausgegangen. Dann erhält man als erstes den Schnittpunkt $P$. Nun entsteht als nächstes das gleichseitige Dreieck $P Q R$, etwa mit Hilfe der bekannten Winkel in (2) (so geschieht es in [1] und [5]; siehe Fig. 6 und Fig. 7), oder mit Hilfe der gleichschenkligen Dreiecke (siehe [8]), welche wir im Beweis von Penrose und Coxeter kennengelernt hatten. Als letztes erscheint dann der dritte Punkt $A$, wobei zum Nachweis dessen Existenz wieder neuer Scharfsinn nötig ist, auf der Bühne. In einem Falle, siehe da, kommt auch „Euclid Bk. III“ zum Einsatz (siehe Fig. 6). 


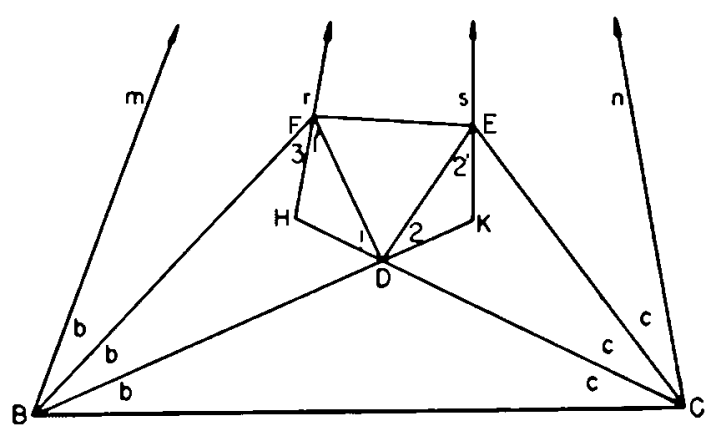

Fig. 7 Beweis von Grossmann (kopiert aus [5])

Diese Beweise sind vielleicht logisch etwas beruhigender, aber dadurch, dass bei verschiedenen Ecken verschiedene Argumentationen gebraucht werden, insgesamt komplizierter.

Danksagung. Der Autor bedankt sich bei dem Referee für wertvolle Verbesserungsvorschläge, bei Ch. Aebi (Genève) für einen wichtigen Literaturhinweis, und bei B. Dudez, Bibliothekar in Genève, für seine grosse Fähigkeit, seltene und seltenste Artikel aufzutreiben.

\section{Literatur}

[1] Child, J.M.: Proof of "Morley's theorem” (by Euclid, Bk. III). Math. Gazette 11 (1923), 171.

[2] Coxeter, H.S.M.: Introduction to Geometry. John Wiley, 1961.

[3] Euclid: The thirteen books of the Elements. Transl. and comm. by Sir Thomas L. Heath, 1925, reprinted Dover 1956.

[4] Geiges, H.: Beweis des Satzes von Morley nach A. Connes. Elem. Math. 56 (2001), 137-142.

[5] Grossmann, H.D.: The Morley triangle: a new geometric proof. Amer. Math. Monthly 50 (1943), 522.

[6] Penrose, R.: Morley's trisector theorem. Eureka 16 (1953), 6-7.

[7] Taylor, F.G.; Marr, W.L.: The six trisectors of each of the angles of a triangle. Proc. Edinburgh Math. Soc. 32 (1913), 119-131.

[8] Venkatachaliengar, K.: An elementary proof of Morley's theorem. Amer. Math. Monthly 65 (1958), $612-613$.

Gerhard Wanner

Section de Mathématiques

Université de Genève

C.P. 240

CH-1211 Genève 24, Schweiz

e-mail: Gerhard.Wanner@math . unige.ch 